\title{
EDUCACIÓN E INTERNET PARA EL DESARROLLO LOCAL
}

Cruz García Lirios ${ }^{\mathbf{1}}$ : Universidad Autónoma del Estado de México. México. garcialirios@yahoo.com

Javier Carreón Guillén: Universidad Nacional Autónoma de México. México. javierg@unam.mx

Daniela Mendoza Alboreida: Universidad Nacional Autónoma de México. México. danielamendozaunam@hotmail.com

José Alfonso Aguilar Fuentes: Universidad Nacional Autónoma de México. México. jorheval@unam.mx

\section{RESUMEN:}

Desde sus inicios, los sistemas educativos formaron parte de un Estado Moderno en el que la liberación de los dogmas religiosos fue la empresa más importante de la Ilustración, aunque se construyó, desde una ética de la recompensa, una nueva homogeneidad del mundo que sometió los saberes y los conocimientos. En la actual sociedad de la información, los dispositivos y tecnologías reducen la liberación del ser a su mínima expresión ya que orientan sus temas de pensamientos, debate y consenso. En este sentido, la importancia de estudiar los procesos que anticipan escenarios de incertidumbre radica en la explicitación de los factores cognitivos desde los que se explica la aceptación de la tecnología y el uso intensivo de los dispositivos electrónicos. Tal ejercicio abrirá la discusión en torno a la globalización digital que se disemina en los procesos educativos y se cristalizan en el aula.

PALABRAS CLAVE: globalización - Internet - dispositivos - tecnología - actitud

\section{EDUCATION AND INTERNET FOR LOCAL DEVELOPMENT}

\section{ABSTRACT:}

${ }^{1}$ Cruz García Lirios: Estudios de Doctorado en Psicología Social y Ambiental, Profesor de asignatura. Autor del Libro: "Cultura y Gobernanza de la Paz Pública". Línea de investigación: Gobernanza y Agenda Sociopolítica de Seguridad

Correo: garcialirios@yahoo.com 
Since its inception, educational systems were part of a Modern State in which the release of religious dogmas was the most important company of the Enlightenment, but was built from an ethical reward, a new homogeneity of the world that submitted the knowledge and skills. In today's information society, devices and technologies reduce the release of the self to a minimum and that direct their thoughts issues, debate and consensus. In this regard, the importance of studying the processes that anticipate scenarios of uncertainty lies in the explicit cognitive factors from which the acceptance of technology and intensive use of electronic devices is explained. Such an exercise will open the discussion on digital globalization that spreads in education and crystallized in the classroom.

KEY WORDS: Globalization - Internet - devices - technology - attitude

\section{INTRODUCCIÓN}

El objetivo del presente estudio es establecer los determinantes de las decisiones e intenciones de uso de Internet para discutir las consecuencias cognitivas. Debido a que los estudios psicológicos de las intenciones y decisiones de uso de Internet parecen circunscribirse a variables cognitivas, es menester elaborar un modelo para establecer las relaciones causales entre los factores tecnológicos, perceptuales, actitudinales e intencionales.

En principio, el uso de Internet ha sido considerado como un conjunto de acciones de compra y venta de productos y servicios a través de protocolos electrónicos o digitales de transferencia de dinero por un bien. En este sentido, el intercambio de información sería fundamental para los objetivos de desarrollo personal. Es decir, las ventajas competitivas en torno a la búsqueda, selección y síntesis de información a través de Internet serían un indicador de las capacidades de manejo y procesamiento de información.

Se trata de la compra y contratación de servicios o productos en los que la percepción de control y las experiencias de consumo explican la consecución de objetivos. Piénsese en la satisfacción del cliente como el resultado de positivas experiencias y altas percepciones de control, las Tecnologías de Información y Comunicación (TIC) estarían determinadas por ambas variables.

En el caso específico de las percepciones, los estudios psicológicos del uso de Internet sostienen que la diversidad de percepciones son las determinantes principales de las decisiones de consumo electrónico y las transferencias de dinero a cambio de un producto o servicio.

A pesar de que existe sustento teórico y empírico para demostrar que las percepciones son factores esenciales en torno a la aceptación, adopción y uso de TIC's, otras variables de orden valorativo, normativo, demográfico, tecnológico, creencial, actitudinal o intencional explicarían en mayor medida el uso de Internet. 
La diversidad de factores predictivos del uso de Internet corresponde con la heterogeneidad de estudios en los que las percepciones, son los factores que más se han matizado para lograr explicar acciones específicas de uso de Internet.

Las percepciones se han dimensionado en cinco aspectos que miden capacidades, habilidades, beneficios, costos, riesgos o retos como expectativas que ocurren en el momento mismo de la decisión de uso de Internet. 0 bien, expectativas que probablemente ocurrirán si los beneficios superan a los costos.

\subsection{Estudios psicológicos del uso de Internet}

Internet es un escenario global en el que la heterogeneidad de identidades y la diversidad de símbolos incide en la construcción de una cultura de rasgos globales, en éstos las desigualdades y expresiones sociales, construyen imaginarios sociales y educativos (Winocur, 2006: p. 532). En este sentido, las redes sociales definidas como promotoras de discrepancias étnicas, religiosas o sexuales en torno a las relaciones cotidianas cara a cara en formas de interacción novedosas mediadas por Internet, principalmente a través de aplicaciones móviles (Sandoval y Saucedo, 2010: p. 133), orientan la frustración y la satisfacción del consumo de productos y servicios (Montero, 2006). La satisfacción de uso de una página web y sus correspondientes aplicaciones y servicios, determinan la experiencia de compra venta de productos y servicios en línea (Hassan, 2006: p. 240). La satisfacción está relacionada directamente con las características sociodemográficas de los usuarios de Internet. Mafé y Blas (2007: p. 139) demostraron el efecto directo e indirecto del sexo, la edad, la formación académica y el salario sobre la compra de productos y la adquisición de servicios. Principalmente, la relación entre el sexo del usuario determinó el consumo en línea. Dicha relación estuvo mediada por la actitud hacia el ciberconsumo. En otro estudio llevado a cabo con 17 variables socioeconómicas, Martínez y De Hoyos (2007: págs. 25-31) demostraron el efecto directo y positivo de la comodidad sobre la motivación de compra en línea. A la luz del factor conveniencia, la comodidad fue el principal determinante de las expectativas de consumo electrónico. En otro modelo, las ofertas fueron predictoras de la motivación de compra y la confianza en el producto, incidió en los motivos de consumo.

El análisis de los determinantes de uso de Internet puede realizarse a partir del Modelo de Aceptación de la Tecnología (TAM por sus siglas en inglés). El TAM incluye variables de orden perceptual, actitudinal e intencional a partir de las cuales se ha demostrado el efecto de variables tecnológicas y organizacionales sobre las intenciones de uso de Internet (Davis, Bagozzi y Warshaw, 1989).

Debido a que los estudios psicológicos han seguido más la incidencia de variables tecnológicas y organizacionales que la influencia de las variables sociodemográficas, los estudios que siguen una trayectoria de explicación a partir del sexo, la edad, el salario o la escolaridad, son escasos (Fuente, Herrero y Gracia, 2010).

No obstante, si las variables perceptuales aluden a procesos cognitivos de eficacia y utilidad en torno al uso de Internet, entonces el tipo de sexo, los años cumplidos, la 
cantidad de salario y el nivel de estudios tendrían una relación directa con los logros y beneficios en torno al uso de Internet en los ámbitos laboral y escolar. Si la percepción de autoeficacia y la percepción de utilidad explican las expectativas de los usuarios al momento de usar Internet, entonces la cantidad de salario y el grado de estudios incentivarían o inhibirían dichas expectativas.

Agarwal (2000: p. 85) plantea que las variables socioeconómicas, sociodemográficas y socioeducativas son consideradas exógenas a la cognición humana y que inciden indirectamente sobre el uso de Internet. En este sentido, los estudios psicológicos de la interacción humanidad-tecnología han especificado relaciones indirectas entre las variables externas y el uso de Internet a través de cogniciones tales como percepciones, actitudes e intenciones (Agarwal y Venkatesh, 2002: p. 169)

Carmel y Agarwal (2002: p. 67) refieren tres niveles de interacción en los que las motivaciones, habilidades y conocimientos se relacionan con Internet. En el primer nivel, los usuarios sólo trasfieren datos mediante Internet. En el segundo nivel, los humanos diversifican su comunicación a través de Internet. En el tercer nivel, llevan a cabo estrategia que implican habilidades y conocimientos para implementar nuevos sistema de producción, distribución y consumo a través de Internet. Este último nivel esta relacionado con la aceptación, implementación, capacitación y producción de ventajas competitivas y estándares de certificación.

El TAM predice que los programas y procesos tecnológico-computacionales determinan variables psicológicas. Hernández, Jiménez y De Hoyos (2007: págs. 2325) demostraron la predicción de diferencias entre los sexos de los usuarios de programas de cómputo con respecto a sus percepciones de facilidad de uso, utilidad y actitud hacia la gestores de venta en línea. Precisamente, el TAM nace con un enfoque técnico en el que se buscada explicar los procesos de transferencia, adopción y sistematización tecnológica computacional. En la medida en que el TAM ha sido desarrollado desde ésta visión técnica que consiste en implementar medidas de eficiencia, eficacia y efectividad sin considerar las diferencias culturales, sociales y personales, el modelo ha ganado prestigio hasta un grado tal que ahora es desarrollado desde múltiples disciplinas. Davis y Venkatesh (1996: p. 20) consideran a los software de Internet como variables exógenas a la aceptación de la tecnología. La relación entre la tecnología y su aceptación implica un proceso tecnológico, racional, deliberado, planificado y sistemático en el que las variables sociotecnológicas tales como capacitación y adiestramiento a partir de una tecnología interactúan con la cognición humana; percepciones, actitudes e intenciones.

Venkatesh, Morris, Davis y Davis (2003: p. 426) advierten sobre el aumento exponencial de los estudios sobre Internet indicado por el incremento hasta de un 50 por ciento en el financiamiento de la investigación organizacional y la consecuente explicación del 40 por ciento de la varianza total en torno a la aceptación y uso de Internet.

En este sentido, es menester explicar las relaciones causales directas e indirectas entre las variables sociodemográficas y la intención de uso de Internet. 
Precisamente, demostrar la relación entre las variables sociodemográficas y las variables psicológicas contribuirá al desarrollo del TAM como un modelo en el que las diferencias sexuales, de edad, educativas y económicas determinarían percepciones, actitudes e intenciones de uso de Internet. En este sentido, el TAM podría desarrollar una teoría explicativa del impacto de las variables tecnológicas sobre la aceptación, adopción y uso de Internet a través los perfiles sociodemográficos.

\subsubsection{Autoeficacia percibida}

En la concepción de Bandura (1977; 1982; 1993; 1994; 1995; 2001) la autoeficacia es una percepción y/o una creencia motivada por ensayos de aciertos y errores personales o impersonales llevados a cabo deliberada o discursivamente. Dado que la autoeficacia alude al fracaso, pero principalmente al éxito, aún a pesar de aquellos ensayos fallidos que incitan al logro, la percepción y creencia de autoeficacia se sustenta en la consecución de objetivos esperados más que en la competitividad, el reconocimiento $\mathrm{o}$ el aprendizaje vicario. Si la autoeficacia es un sistema de percepciones y creencias enfocada al éxito, entonces el grupo al que pertenece o quiere pertenecer el agente autoeficaz, está relacionado con el éxito. Debido a que los grupos son diversos, la autoeficacia varía en función de esta diversidad. Un grupo competitivo atribuye éxito a uno de sus integrantes cuando éste ha sobrepasado los logros antecedentes que por cierto estaban fijados por el grupo. En este sentido, el concepto de autoeficiencia parece fehacientemente ajustado a la influencia de un grupo sobre los objetivos, el sistema y logros de un individuo.

Si la autoeficacia es un sistema de percepciones que incentivan los logros delimitando las capacidades eficaces, la autoeficiencia también sería un sistema de percepciones y creencias, pero a diferencia de la autoeficacia, éstas estarían orientadas a la ejecución de un procedimiento o tecnología. Los factores que impulsan la autoeficacia serían idénticos en el caso de la autoeficiencia. Si la competitividad, el reconocimiento y el aprendizaje vicario impulsan la autoeficacia, entonces la autoeficiencia también tendría ese impulso.

\subsubsection{Autoeficiencia computacional}

Los estudios de la autoeficiencia computacional han establecido relaciones asociativas y causales entre percepciones, creencias, actitud, experiencia, conocimiento, habilidades, confort, ansiedad, utilidad, afectividad, control y facilidad percibida.

Busch (1995) estableció la predicción de la autoeficacia computacional a partir de la experiencia previa. Este hallazgo concuerda con el estudio de Khorrami (2001) en el que demostró una asociación positiva y significativa entre las creencias de control y la disposición a teclear. Particularmente, Durndell y Haag (2002) han relacionado la autoeficiencia con el aumento de la ansiedad al momento de manejar el ordenador. Barbeite y Weiss (2004) predijeron el confort computacional y el ciber-confort a partir de la eficiencia computacional y los avances en la capacidad percibida. Hsu y Chiu (2004) demostraron el efecto exógeno de la ciber autoeficiencia mediante el 
ajuste de un modelo estructural. En dicha estructura, el uso de una tecnología esta determinado por el efecto de la ciber autoeficiencia a través de la intención y la actitud hacia la tecnología (Compeau y Higgins, 1995). Se trata de una estructura que explica un proceso deliberado, planificado y sistemático de adopción, aceptación y ciber-uso. Jonhson y Wardlow (2004) encontraron que la autoeficiencia asociada con la ansiedad en el uso computacional incrementaba la predicción y la significancia del uso de Internet. Sam, Othman y Nordin (2005) establecieron diferencias significativas entre las actitudes hacia Internet, la autoeficiecia computacional y la ciber-ansiedad con respecto a habilidades de almacenamiento de información. Chu (2006) al demostrar que la percepción de eficiencia variaba en función del uso previo de la computadora demostró que la percepción de logro estaba determinada por el número de ensayos en torno al manejo del computador. Torkzadeh, Chang y Demirhan (2009) demostró diferencias individuales significativas entre hombres y mujeres que recibieron adiestramiento y registraban ansiedad y actitud hacia la ciber-navegación con respecto a la autoeficiencia computacional y la ciberautoeficiencia. Wu y Tsai (2006) encontraron una asociación entre la efectividad y la autoeficiencia.

No obstante, los estudios psicológicos han consolidado el efecto directo de la autoeficiencia sobre la percepción de utilidad. Rose y Fogarty (2006) demostraron la influencia de la autoeficiencia percibida sobre la percepción de utilidad que junto con la actitud hacia la tecnología y la intención de uso, transfirieron los efectos de la autoeficiencia en el manejo de Internet. Acorde con este resultado, Reid y Levy (2008) establecieron la incidencia mediadora de la percepción de utilidad y las actitudes hacia la tecnología en la relación causal indirecta entre la autoeficiencia computacional sobre el uso de la tecnología. Ambas variables endógenas mediadoras incrementaron el efecto de la autoeficiencia percibida. Bennet, Zimmer, Gundlach y McKnight (2008) encontraron, mediante un modelo estructural, la incidencia de la internalización y la externalización sobre la ciber-capacidad percibida. El ajuste de la estructura se demostró con cuatro muestras diferentes. No obstante, la covarianza entre ambos factores exógenos es cercana a la unidad lo cual limita su validez discriminante. Paraskeva, Bouta y Papagianni (2008) demostraron que las habilidades básicas, los avances de las habilidades y el manejo de datos están asociados con la eficiencia computacional. Un incremento en el manejo de la computadora, los archivos y la captura de datos aumentaban la percepción de eficiencia. Reid y Levy (2008) demostraron, mediante un modelo de trayectorias, la predicción indirecta de la intención de uso a partir de la autoeficiencia computacional. En esta trayectoria de predicción, la percepción de utilidad, facilidad de uso percibida y actitud hacia la computación transmitieron significativamente el efecto de la ciber-capacidad percibida. Vekiri y Chronaki (2008) encontraron que la ansiedad, en el caso de las mujeres y la frecuencia de uso, en el caso de los hombres, están relacionados directa, positiva y significativamente con la autoeficiencia computacional. Por su parte, Sadeé y Kira (2009) demostraron el efecto mediacional de la ansiedad computacional sobre la percepción de utilidad a través de la autoeficiencia computacional. En un primer estudio, la ansiedad computacional tenía una relación directa, negativa y significativa sobre la percepción de utilidad. Al incorporar la variable de autoeficiencia computacional, el efecto 
indirecto se convertía en positivo y significativo. A partir de este principio se infirió la incidencia de la autoeficiencia computacional sobre el uso de la tecnología. Saadé y Kira (2009) establecieron en un modelo mediacional el efecto directo e indirecto de la ciber ansiedad sobre la percepción de utilidad. Dicho efecto, también se incrementaba a través de la autoeficiencia computacional. Teh, Chong, Yong y Yew (2010) plantean que los logros configuran avances de autoeficiencia los cuales determinan el nivel de conocimiento. Finalmente, Chuo, Tsai, Lan y Tsai (2011) explicaron la relación directa, positiva y significativa entre la Cibereficiencia y la Ciberutilidad como determinantes indirectos y directos de la decisión de usar Internet. Simsek (2011) encontró que un incremento en los valores de la capacidad percibida incide en un aumento de los valores intencionales regulados por los valores perceptuales. Se trata de un efecto indirecto en el que las creencias de eficiencia interactúan con la ansiedad (Ademola, 2009).

La relevancia de los avances en las habilidades básicas es otro determinante de la autoeficiencia computacional. En la medida en que las habilidades de manejo de archivos y datos se desarrollan propician un incremento en los conocimientos y las capacidades percibidas (Liang y Tsai, 2008). La inclusión de la autoeficiencia computacional en la extensión del TAM explicó la relación entre la autoeficiencia educativa y la utilidad de Internet. En la medida en que los usuarios incrementan sus experiencias de uso de Internet también aumentan sus expectativas, necesidades, oportunidades y exigencias de uso de Internet (Meléndez y Moreno, 2006). Esto es, los estudiantes y académicos no sólo utilizan Internet para fines educativos sino además comerciales. En este sentido, la diversificación de Internet sería explicada por la multiplicidad de sus funciones que al ser utilizadas por los usuarios activan la creatividad individual y la innovación grupal potenciales para el crecimiento heterogéneo de Internet.

\subsubsection{Utilidad percibida}

Los estudios perceptuales tecnológicos han demostrado un efecto indirecto y directo, positivo y negativo, de las percepciones sobre el uso de las tecnologías. A través de las actitudes e intenciones, las percepciones de facilidad de uso, utilidad y control se han erigido como las determinantes de las decisiones y manejo de tecnologías. La percepción de facilidad de uso y la percepción de utilidad han sido planteadas como los determinantes de la aceptación de la tecnología.

Davis (1989: p. 320) define la facilidad de uso como el grado de información en torno a la inversión de esfuerzo para manejar una tecnología. Respecto a la percepción de utilidad, la define como el grado de información en torno a los beneficios esperados por el manejo de una tecnología.

Davis (1993) estableció el efecto indirecto de la percepción sobre el uso de la tecnología. Tanto la percepción de facilidad de uso como la percepción de utilidad afectan indirectamente la aceptación de tecnologías. En esta trayectoria causal, las actitudes hacia la tecnología son mediadoras del efecto perceptivo. Lee, D., Park, J. \& Ahn, J. (2001) demostraron el efecto de las percepciones sobre el uso de la 
tecnología. La tecnología percibida como fácil de usar afecta indirecta y directamente su uso. A través de la percepción de utilidad, la facilidad de uso percibida tiene una incidencia indirecta que impacta el uso de la tecnología con mucho mayor significancia en relación a su efecto directo. Es decir, los individuos que perciben una habilidad para usar la tecnología incrementan sus expectativas con los beneficios que la tecnología les puede llegar a otorgar. Venkatesh, Morris, Davis y Davis (2003) establecieron, con una muestra de voluntarios de la industria de entretenimiento y telecomunicaciones y operarios de bancos y administración tributaria, la predicción de la intención de uso tecnológico a partir de las percepciones de facilidad de uso, utilidad, control y autoeficiencia. Cuando la percepción de control interactúa con la edad provocan un efecto positivo sobre la intención de uso. Respecto a la predicción del uso de tecnología, la percepción de control es la variable que causa un mayor efecto. Pin (2004) extendió los límites de la percepción a plantear la percepción de ejecución como el blanco principal de la relevancia de tecnología. Es decir, si en un contexto dado una tecnología se hace relevante, ésta activa procesos perceptuales que predicen su uso. A través de esta trayectoria, la facilidad de uso, utilidad y actitud son intermediarios del efecto contextual sobre el uso de la tecnología. Lai y Lee (2005) extendieron la predicción de las actitudes hacia la tecnología y las decisiones de consumo en tres grupos clasificados como no adoptantes, adoptantes y neutros. En los tres grupos establecieron la predicción de la percepción de beneficios sobre las disposiciones favorables a la tecnología y sus decisiones de adopción. Incluso, establecieron la relación causal entre la percepción de facilidad de uso sobre la utilidad percibida. Lim, Lim y Heinrichs (2005) establecieron la predicción de la compra electrónica a partir de percepciones de seguridad y el disfrute percibido. En este modelo, la percepción de facilidad de uso resultó ser un mejor mediador en comparación con la percepción de utilidad. En otro modelo, la utilidad percibida como mediadora incrementa el efecto de las otras tres percepciones. En un tercer modelo, la facilidad de uso percibida es mejor predictora en comparación con las otras tres percepciones relacionadas directamente. Porter y Donthu (2006) encontró que la edad y la educación determinan indirectamente el uso de la tecnología a través de la percepción de facilidad y la actitud hacia la tecnología. En la medida en que la edad y el nivel de estudios se incrementan, las expectativas y disposiciones aumentan el uso de la tecnología.

No obstante el poder predictivo de las percepciones, Limayen, Hirt y Cheung (2007) plantearon una relación directa entre la percepción de utilidad y el uso continuo de sistemas de información. Esta relación estuvo moderada por la satisfacción posterior al uso de tecnología y la intención de volver a usar la tecnología. Herrero, Rodríguez y Trespalacios (2006) establecieron la influencia de la percepción de utilidad sobre la actitud y la intención de compra. Sólo la percepción de facilidad de uso de la tecnología tuvo un efecto indirecto sobre las decisiones de compra a través de las actitudes. Chin (2009) comparó los efectos indirectos con efectos directos de la percepción sobre la intención de uso. Encontró que los efectos directos son más significativos que los indirectos de las percepciones de utilidad y facilidad sobre la intención de uso de la tecnología. Hee y McDaniel (2011) demostraron la predicción de las decisiones de adquisición y uso de tecnologías a partir de las percepciones de utilidad. Es decir, los beneficios percibidos por la compra de tecnologías inciden 
directa, positiva y significativamente sobre su uso. En la medida en que los clientes y usuarios perciben más beneficios que riesgos, consumen tecnología. En los estudios perceptuales tecnológicos la utilidad y la facilidad de uso son los factores determinantes de las intenciones y uso de tecnologías. La evolución de Internet influyó en el desarrollo de los estudios perceptuales tecnológicos. La percepción de utilidad y facilidad de uso ahora son factores que explican el uso de Internet.

Davis (2006: p. 400) realizó un metanálisis en el que demostró la relación causal entre la percepción de utilidad y la percepción de facilidad de uso de la tecnología como los determinantes de la aceptación de la tecnología. Principalmente, la utilidad percibida es considerada la predictora por excelencia de la aceptación y uso de la tecnología. Sin embargo, la percepción de utilidad sólo es transmisora, intermediaria o mediadora del efecto de las variables externas o exógenas sobre el comportamiento humano.

Las variables externas tales como el sexo, el nivel educativo y el ingreso económico familiar al interactuar propician un efecto moderador sobre la aceptación y uso de la tecnología. Dicha relación causal al ser indirecta, estaría mediada por la percepción de utilidad. Sin embargo, sólo tres estudios han llevado a cabo la especificación y demostración de un modelo en el que las variables sociodemográficas interactúan para predecir el uso de la tecnología a través de las variables perceptuales tales como la utilidad y la facilidad de uso. Tales son los caso de las investigaciones llevadas a cabo por D'ambra y Wilson (2004), Porter y Donthu (2006), Bigne, Ruíz y Sanz (2007) en los que establecieron la incidencia de las variables sociodemográficas del sexo y la edad sobre el uso de Internet. En la medida en que se incrementaba la edad de los usuarios, aumentaba su percepción de utilidad y su aceptación por la tecnología.

No obstante, los estudios psicológicos de la interacción humanidad con tecnología y cognición con Internet han ignorado el poder predictivo del ingreso económico familiar, la renta personal, el estatus económico, la calidad de vida, el bienestar subjetivo y el nivel educativo.

\subsubsection{Actitudes hacia el comportamiento}

Los estudios de las actitudes hacia el comportamiento se han enfocado en su ambivalencia. Briñol, et, al., (2004: p. 373) señalan que las personas tratan de equilibrar la información favorable y desfavorable hacia ese objeto disposicional manteniendo actitudes ambivalentes. Es decir, los objetos actitudinales son parte del entorno en el que se encuentran las personas y su necesidad de ordenarlo, predecirlo y controlarlo (Orantes, 2011). Por ello, aunque el objeto actitudinal sea consistente con sus percepciones, valores y creencias, las personas deben contrastar dichos objetos con los comportamientos asociados a ellos. En tanto escenario de compra y venta de productos y servicios, Internet propicia actitudes positivas y negativas en los usuarios. Si las personas tienen el poder adquisitivo para comprar un objeto o adquirir un servicio pueden no tener la confianza y seguridad suficientes para encargar el bien a cambio de transferir el equivalente de su precio en dinero 
(López y López, 2011). Esto inhibe el comercio electrónico ya que el displacer propicia desconfianza. En la medida en que una tecnología es poco accesible, propicia emociones negativas que desmotivan al cliente potencial.

\subsubsection{Actitudes hacia el uso de Internet}

Los estudios de actitudes hacia Internet han demostrado las relaciones asociativas y causales entre las habilidades, ansiedades, normas, percepciones, intenciones y comportamientos. En la investigación de Dishaw y Strong (1999) las actitudes son intermediarias del efecto de las percepciones sobre la intención y el uso de la tecnología. En otro modelo estructural, las actitudes regulan la influencia de las habilidades de ajuste de una tecnología a la función del trabajo y la experiencia de uso de la tecnología. Amoako y Salam (2004) midieron el impacto de un adiestramiento tecnológico sobre su uso. Dicho efecto, estuvo regulado por las percepciones, actitudes e intenciones. No obstante, la actitud parece no transmitir el efecto de la autoeficiencia computacional. En el modelo estructural de Gong, Xu y Yu (2004) la actitud regula la influencia de la facilidad y la utilidad percibida, pero la capacidad percibida tiene un efecto directo. En un estudio correlacional Shih (2004) demostró la asociación positiva y significativa entre la actitud y la percepción. Las disposiciones hacia la tecnología estaban relacionadas directamente con la utilidad y la facilidad percibida. En otro estudio Lai y Lee (2005) demostraron la robustez del TAM con diversos subgrupos. Las actitudes asociadas a las percepciones presentaron mínimas variaciones en las sub-muestras. Tanto expertos como neófitos arrogaron respuestas muy similares lo que llevó a inferir la verosimilitud del modelo de medición y la demostración de las hipótesis en el modelo estructural.

Este fue el caso de la investigación de Liu, Liao y Peng (2005) quienes incluyeron variables perceptuales de utilidad y facilidad como determinantes de la actitud la cual incidió en la intención de uso de Internet. En este modelo estructural, la actitud transmite los efectos de la percepción sobre la intención. Un incremento en las expectativas de uso determina indirectamente la probabilidad de usar la tecnología de información a medida que las disposiciones evaluativas regulan la tendencia de utilidad. En contraste, en el modelo estructural de Porter y Donthu (2006) el efecto de las variables socioeconómicas y demográficas sobre el uso de Internet, las actitudes son determinantes endógenas de primer orden. Es decir, la edad, educación y raza tienen una determinan indirectamente el uso de la tecnología de información a través de percepciones y actitudes. En la medida en que las muestras se diferencian por su edad, raza y educación, provocan un efecto sobre la utilidad y facilidad percibidas que a su vez inciden en las disposiciones favorables hacia Internet y su posterior uso. Shepers y Wetzelts (2007) realizaron un meta-análisis de las investigaciones en torno al efecto de la norma subjetiva sobre el uso de la tecnología. Dicho impacto, regulado por las percepciones, actitudes e intenciones, fue demostrado en diferentes muestras, con distintas tecnologías y múltiples valores culturales. En un estudio diferente, Bertrand y Bouchard (2008) demostraron la asociación entre las actitudes y las motivaciones, habilidades, ansiedades y percepciones. Establecieron correlaciones con variables diferentes a las utilizadas en el estado del arte. Se trata de las percepciones de control y autoeficiencia que junto 
a la norma subjetiva y la ansiedad enriquecen el TAM. Ha y Stoel (2009) modificaron el TAM al agregarle la percepción de transacción en la banca electrónica. En este modelo las actitudes transmitieron el efecto de las percepciones sobre la intención de transferencia. Shroff, Deneen y $\mathrm{Ng}$ (2011) demostraron la vigencia de la actitud como variable mediadora de las percepciones. Puesto que la facilidad modera el efecto de la utilidad percibida, la actitud transfiere su influencia al uso de Internet.

Esto demuestra la verosimilitud de las relaciones hipotéticas del TAM. En la medida en que la gente percibe a la tecnología como un objeto accesible y benéfico es más proclive a aceptarla y consumirla vía sus evaluaciones. En efecto, la actitud hacia Internet es transmisora de las percepciones y antecedente de la intención que es la determinante del uso de Internet.

Las variables sociodemográficas tales como el sexo, la edad y el salario son determinantes indirectos de la actitud hacia la tecnología, Internet, comercio y consumo electrónico.

D'ambra y Wilson (2004: p. 305) establecieron una relación indirecta entre la edad y el uso de la tecnología. A través de la utilización de la tecnología se observó un incremento de la relación entre la variable dependiente y la variable independiente. Cabe señalar que ninguna de las variables del TAM estuvo implicada en el hallazgo. Sin embargo, es de suma importancia considerar el efecto indirecto y positivo ya que es un indicio de una probable estructura de relaciones asimétricas entre las edades de los usuarios la que propiciaría el uso sistemático de una tecnología. En el TAM, la edad es una variable externa y la actitud una variable interna que explica el uso de la tecnología en interacción con las percepciones e control y utilidad. En este modelo, la edad es una característica individual que incide en las habilidades tecnológicas. La relación entre ambas variables explica la actitud hacia el uso de una tecnología en el trabajo.

Porter y Donthu (2006: p. 1001) demostraron el efecto indirecto del nivel de estudios sobre el uso de la tecnología a través de la percepción de facilidad de uso y la actitud hacia la tecnología. En la medida en que se incrementan los valores de la variable sociodemográfica incidían en los valores de la variable conductual. El efecto indirecto fue transmitido por la facilidad de uso percibida y la disposición positiva hacia la tecnología. A través de la percepción de utilidad y la facilidad de uso percibida demostraron efectos incrementales de las variables sociodemográficas consideradas exógenas por el TAM. A pesar de que existe una relación directa, negativa y significativa entre las variables sociodemográficas y las percepciones de la tecnología, las actitudes reciben un efecto indirecto negativo. En la medida en que la edad, la educación y la renta se incrementan disminuyen sus percepciones afectando positivamente la actitud hacia la tecnología. Es decir, tanto la utilidad como la facilidad de uso percibidas se incrementan a medida que el usuario es más joven, su nivel de instrucción es básico y su ingreso es mínimo provocando un aumento en sus creencias hacia la tecnología. A la luz del TAM, la relación indirecta entre el nivel de estudios y el uso de la tecnología fue un hallazgo de suma relevancia ya que sólo las variables externas tales como la implementación de un programa tecnológico o 
sistema computacional producían un efecto indirecto. El resultado, demuestra que la selección de las variables sociodemográficas puede servir en la implementación de programas y estrategias tecnológicas, computacionales, informacionales, relacionales y administrativas.

Bigne, Ruíz y Sanz (2007: p. 56) establecieron la predicción de la intención de uso de Internet a partir de una variable sociodemográfica como la edad y a través de una variable mediadora por excelencia como lo es la actitud hacia el uso de la tecnología o sistema de información. Sin embargo, demostraron una relación indirecta, positiva y significativa entre la edad y el consumo móvil. Se trata de un modelo que explica la adopción del comercio móvil en el que se predice el consumo futuro de productos y servicios utilizando un teléfono inteligente.

\subsubsection{Actitudes hacia el uso de internet en América Latina}

En el contexto de América Latina, sólo siete estudios han demostrado la confiabilidad, validez de la actitud hacia Internet así como su relación causal con percepciones de utilidad y facilidad de uso e intenciones de aceptación, adopción, uso, compra o adquisición de productos y servicios en protocolos de transferencia digital de datos. En este sentido, la actitud se ha empleado como una variable mediadora y transmisora del efecto perceptual sobre la intención de uso. Estos estudios corroboran el supuesto de las teorías y modelos de acción razona, comportamiento planificado y aceptación tecnológica en torno al cual las actitudes son componentes de una estructura o sistema cognitivo conductual en el que los individuos sistematizan sus expectativas, disposiciones, decisiones y acciones en torno a un objeto que en este caso es tecnológico.

Bonilla y Bonilla (2006: p. 105) demostraron la asociación entre las actitudes y las percepciones de utilidad y facilidad de uso y las intenciones de uso. En la medida en que las expectativas se incrementaban también aumentaban los valores disposicionales los cuales a su vez incidían en las decisiones de uso de Internet.

Mafé y Blas (2007: p. 139) demostraron la relación causal entre la actitud hacia la exposición y compra de productos. A través de la exposición de productos en televisión, las actitudes incidieron sobre la adquisición de los mismos. El efecto directo entre la actitud y la compra fue menor en comparación a la mediación de la exposición del producto.

Martínez y De Hoyos (2007: p. 26) establecieron tres dimensiones actitudinales para demostrar sus efectos sobre la motivación de compra. La comodidad, la oferta y el conocimiento incidieron directa, positiva y significativamente sobre los motivos de las personas al momento de adquirir o comparar un producto o servicio en protocolos de transferencia de datos e intercambio de bienes.

Sanz, Mafé y Aldás (2008: p. 63) demostraron las relaciones causales entre las actitudes y las percepciones e intenciones. Las utilidad y la facilidad de uso percibidas incidieron directa, positiva y significativamente sobre las actitudes hacia el 
uso de Internet. En este sentido, las decisiones de uso de Internet fueron predichas por las disposiciones hacia la tecnología.

Suárez, García y Álvarez (2008: p. 15) establecieron diferencias significativas entre los usuarios de encuestas en línea con respecto a sus actitudes hacia dichos instrumentos. Evaluaron seis ítems relativos a la facilidad, disfrute, valoración, extensión, tiempo e intención de las encuestas en línea. En todos y cada uno de los ítems se encontraron diferencias significativas entre los respondientes y sus disposiciones al momento de contestar una encuesta en Internet.

Hernández, Robles y Mella (2009: p. 34) establecieron diferencias significativas entre sexo, edad y tipo de tecnología con respecto a las actitudes hacia el ordenador y el servicio de Internet. Los tipos de ordenadores, al momento de su uso, fueron evaluados positivamente por los usuarios del mismo modo que los servicios de Internet, transferencia e intercambio de datos. Las diferencias entre niveles, nulo, básico, intermedio, avanzado y experto fueron significativas en relación a las disposiciones positivas hacia el uso de Internet.

En síntesis, las disposiciones hacia el uso de una tecnología como Internet tienden a ser positivas en la medida en que los usuarios o clientes potenciales incrementan sus expectativas de utilidad y facilidad de uso. Es decir, si las personas se perciben como capaces de usar y aprovechar una tecnología, entonces sus disposiciones serán favorables motivando sus decisiones de consumo.

No obstante, los estudios psicológicos del sistema o estructura cognitiva -conductual en torno a Internet, no han incorporado la ambivalencia actitudinal en torno a la cual las disposiciones se verían afectadas por las discrepancias perceptuales tales como; altas expectativas de un producto o servicio y bajos niveles de satisfacción posterior a su uso. En otras palabras, la tendencia de las personas a comparar sus experiencias e informaciones previas con tecnologías en relación a innovaciones computacionales y su prevalencia a equilibrar las discrepancias entre lo que esperan de una tecnología y sus niveles de satisfacción, parece estar determinada por sus expectativas y habilidades en torno al uso de Internet. Este mismo efecto incidiría en sus decisiones de compra y venta de productos y servicios.

\subsection{Determinantes de la intención de uso de internet}

EI TAM refiere al proceso de adopción, aceptación y sistematización de información a través del uso de Internet. Dicho proceso es diferente al de adicción o apego a una tecnología en el cual el usuario se adhiere a ella compensando una ausencia personal o laboral. Es decir, las personas adictas se alejan de su entorno cotidiano para sumergirse en su entorno virtual el cual modificará sustancialmente su estilo de vida (Beranuy, Chamarro, Graner y Carboell 2009: p. 481). Tal es el caso del cibersexo que en el estudio de Ballester, Gil, Gómez y Gil (2010: p. 1051) establecieron asociaciones significativas con las horas en línea, la visita a páginas pornográficas, la frecuencia de cibersexo y las horas dedicadas a esta adicción. Este hallazgo es de suma relevancia si se considera que la compra venta de productos y servicios en 
Internet esta determinada por factores psicológicos muy similares a los que operan en el comercio cara a cara, pero que a diferencia de éste, el comercio electrónico es más incierto por el desconocimiento tecnológico computacional el cual se erige como una barrera del consumo electrónico. En este sentido, Ruíz y García (2007: p. 56) demostraron que la decisión de compra en Internet es determinada por la satisfacción con el producto, la desconfianza en la organización y la utilidad percibida. En efecto, como ocurre en el comercio cara a cara, la satisfacción es un factor preponderante para el consumo electrónico que agregado a la percepción de riesgo, desconfianza o incertidumbre incrementan las expectativas de oportunidad, rendimiento y mejora de la calidad de vida de los consumidores. Por su parte, Lorenzo, Constantinides, Gómez y Geurts (2008: p. 59 y 62) demostraron el efecto directo, positivo y significativo de la usabilidad, interactividad, confianza, estética y mercadotecnia sobre el consumo virtual.

\subsubsection{Intención de uso de Internet}

La intención es una variable psicológica transmisora de los efectos de las habilidades, adiestramientos, normas, percepciones y actitudes hacia Internet sobre el consumo electrónico. Dishaw y Strong (1999) establecieron tres modelos de trayectorias en los que la decisión de consumo electrónica fue transmisora de lo efectos perceptuales y las habilidades sobre el uso tecnológico. En el estudio de Amoako y Salam (2004) desarrollaron un programa de adiestramiento en el que indujeron creencias utilitarias grupales. Los resultados muestran que las habilidades de adiestramiento, información y comunicación tuvieron un efecto indirecto sobre las decisiones de compra a través de Internet. En dichos modelos, las percepciones fueron trasmisoras endógenas de segundo orden y las actitudes hacia la tecnología fueron transmisoras endógenas de primer orden. En este sentido, Gong, Xu y Yu (2004) establecieron el efecto de las habilidades y las percepciones sobre la intención de uso. Las actitudes fueron las transmisoras de la facilidad y utilidad percibida sobre las decisiones de implementación. Klopping y McKinney (2004) la facilidad y la utilidad percibida fueron determinantes directos de la decisión de consumo electrónico. Liu, Liao y Peng (2005) demostraron el efecto directo y significativo entre la utilidad percibida y las actitudes sobre la intención de compra.

Al agregar el ajuste de la tecnología a la tarea en el segundo modelo hipotético, se logró demostrar un efecto indirecto de las percepciones sobre el consumo a través de las intenciones de compra. No obstante, la inclusión de otras percepciones en el modelo de medición, la utilidad percibida fue la determinante de las ciber-decisiones. Roca, Chiu y Martínez (2007) demostraron una serie de hipótesis complejas y sin precedentes en un modelo estructural en el que las influencias personales, los sistemas de información y las habilidades de uso fueron las variables exógenas que afectaron indirectamente las decisiones aprendizaje en Internet. Otro factor relevante fue la satisfacción como la variable endógena de primer orden y las percepciones como endógenas de segundo orden quienes transferían los efectos de las variables exógenas mencionadas. Saadé, Nebebe y Tan (2007) demostraron la predicción indirecta y directa de la intención de compra a partir de las percepciones. 
En tal modelo, las actitudes fueron transmisoras de dichos efectos y la percepción de utilidad fue la determinante directa. Shepers y Wetzels (2007) establecieron un modelo de trayectorias en el que las percepciones y las normas tuvieron efectos indirectos sobre el consumo electrónico. Las actitudes y las decisiones fueron mediadoras de dichos efectos. En el modelo, las percepciones y las normas determinaron directamente a las intenciones de uso de la tecnología de información. La utilidad percibida fue el determinante esencial de las ciber-decisiones. La investigación de Wu, Shen, Lin, Greenes y Bates (2008) demostró un modelo de trayectorias en el que las percepciones, normas y transacciones fueron incluidas para predecir la intención de consumo electrónico. En este modelo la norma personal fue la transmisora del efecto de un adiestramiento sobre las ciber-decisiones. En un modelo diferente a los citados, Chang (2009) estableció el efecto de dos sistemas informáticos sobre la satisfacción en torno al uso de una tecnología de información en la que las percepciones fueron mediadoras o transmisoras de los efectos informáticos y la utilidad fue la determinante directa, positiva y significativa principal. En un relevante estudio llevado a cabo por Ha y Stoel (2009) demostraron la trayectoria de incidencia de la calidad electrónica de compra sobre la intención de consumo electrónico. En este modelo las percepciones y actitudes resultaron transmisoras de los estándares de calidad sobre las decisiones de compra en Internet. Finalmente en el trabajo de Shrrof, Denenn y $\mathrm{Ng}$ (2011) replicaron el TAM y establecieron un efecto indirecto de las percepciones sobre la intención de adopción de tecnología. En este modelo, la decisión de consumo fue una variable endógena de primer orden que transmitió los efectos perceptuales sobre el uso de la tecnología.

En síntesis, la intención o decisión de consumo electrónico es transmisora de experiencias, normas, percepciones y actitudes previas con el uso de Internet. El estado del arte muestra que las intenciones se han consolidado como las determinantes directas, positivas y significativas de la adopción, implementación y desarrollo de tecnologías. La intención de compra, la intención de uso y la intención de sistematización de una tecnología es la variable transmisora por excelencia de los efectos externos sobre el uso de una tecnología.

No obstante, los estudios psicológicos del uso de la tecnología incluyen en sus modelos teóricos y modelos estructurales sólo la relación entre la intención y el uso de la tecnología soslayando el poder predictivo de las variables sociodemográficas tales como; el sexo, la edad, el nivel de estudio y el ingreso económico familiar. Sólo en los estudios de D'ambra y Wilson (2004), Porter y Donthu (2006) y Bigne, Ruíz y Sanz (2007) las variables sociodemográficas de la edad fueron establecidas como predictoras de la intención a través de las percepciones y las actitudes. Más aún, el estado del arte ha excluido la interacción entre las variables sociodemográficas en relación causal directa, positiva y significativa con la intención conductual. Es decir, el efecto moderador del sexo, el ingreso económico familiar y el nivel educativo sobre la intención de uso de la tecnología ha sido soslayado en la TAM.

\section{OBJETIVOS}

Establecer los ejes de discusión y temas de consenso en torno al establecimiento de 
una agenda en materia de privacidad y uso intensivo de dispositivos electrónicos orientados al Desarrollo local.

\section{METODOLOGÍA}

Se llevó a cabo un estudio documental en torno a la aceptación, adopción y uso de Internet con una muestra de 93 fuentes documentales seleccionadas a partir de registro DOI e indexadas desde 2010 a 2014 con las palabras claves de Internet y Desarrollo Local. La información fue buscada en las bases de datos de LATINDEX, REDALYC, DIALNET, SCOPUS, COPERNICUS y SCIELO.

\section{RESULTADOS}

A partir de los hallazgos revisados, se propone un modelo para explicar las relaciones de dependencia entre las variables de un modo integral.

Actitud hacia el uso de Internet. Son asociaciones entre creencias (información disponible) y experiencias de uso de Internet (Aqarwal y Venkatesh, 2002). Por ejemplo, considérese: "En Internet será posible encontrar los productos que busco, pero sólo yo sé en dónde encontrarlos más baratos". Tal aseveración se califica con siete opciones de respuesta que van desde "totalmente de acuerdo" hasta "totalmente en desacuerdo".

Creencias en torno al uso de Internet. Se refiere a la información específica sobre los contenidos de Internet considerando protocolos de compra, venta o trueque de productos y servicios a través de transferencias bancarias o convencionales. Tal cantidad de información puede ser generada por cualquier fuente siempre y cuando el usuario la utilice para un propósito deliberado, planificado y sistemático de consumo (Agarwal, 2000). Por ejemplo, considérese: "En Internet están todos los servicios y productos que un consumidor como yo está buscando". Tal aseveración se evalúa como "falsa" o "verdadera" por parte del encuestado.

Intención de uso de Internet. Son decisiones de compra, venta o intercambio de productos y servicios a través de protocolos electrónicos de transferencia de dinero o cualquier otro equivalente (Carmel \& Agarwal, 2002). Por ejemplo, considérese: "En amazon.com compraría un artículo científico especializado." Tal aseveración se evalúa con cuatro opciones de respuesta que van desde "siempre" hasta "nunca".

Percepción de control en torno al uso de Internet. Son expectativas relativas al uso conveniente de Internet para lograr algún objetivo determinado (Davis, 1989). Por ejemplo, considérese: "Internet es un universo de información en el que puedo seleccionar información actualizada". Tal aseveración se evalúa con cuatro opciones de respuesta que van desde "siempre" hasta "nunca".

Percepción de eficacia en torno al uso de Internet. Son expectativas alusivas al logro 
de búsqueda, selección y procesamiento de información para fines personales (Davis, 1993). Por ejemplo, considérese: "En google académico podré encontrar información especializada que aumentará mis ventajas competitivas". Tal aseveración se evalúa con cuatro opciones de respuesta que van desde "muy probablemente" hasta "muy improbablemente".

Percepción de eficiencia en torno al uso de Internet. Se refiere a la búsqueda, selección y síntesis de información a través de una plataforma o interfaz de Internet (Davis, 2006). Por ejemplo, considérese: "En Dialnet puedo realizar una búsqueda de información sin palabras claves". Tal aseveración se evalúa con cuatro opciones de respuesta que van desde "siempre" hasta "nunca".

Percepción de facilidad de uso de Internet. Se refiere a las expectativas de manejo sistemático de Internet (Venkatesh, Morris, Davis \& Davis, 2003). Por ejemplo, considérese: "Puedo usar la interfaz de mi banca electrónica cada vez que quiero comprar un artículo". Tal aseveración se evalúa con cuatro opciones de respuesta que van desde "siempre" hasta "nunca".

Percepción de riesgo en torno al uso de Internet. Son expectativas de costos superiores a los probables beneficios por la compra o venta de productos o servicios a través de protocolos digitales de comercio (Lee, Park \& Ahn, 2001). Por ejemplo, considérese: "Transferiría dinero a través de la banca electrónica si ésta impidiera la intromisión de hackers". Tal aseveración se evalúa con cuatro opciones de respuesta que van desde "muy probablemente" hasta "muy improbablemente".

Percepción de utilidad en torno al uso de Internet. Son expectativas de mayores beneficios en referencia a los costos de tiempo, dinero y esfuerzo al momento de llevar a cabo una búsqueda de información, producto o servicio específico (Davis, 2006). Por ejemplo, considérese: "En facebook podré charlar con comunidades científicas especializadas". Tal aseveración se evalúa con cuatro opciones de respuesta que van desde "muy probablemente" hasta "muy improbablemente"

Uso de Internet. Son acciones de compra, venta o intercambio de productos, servicios o cualquier otro bien tangible e intangible a través de protocolos electrónicos (Venkatesh, Morris, Davis \& Davis, 2003). Por ejemplo, considérese: "Intercambio música con mis contactos de facebook.com en:". Tal aseveración se evalúa con cuatro opciones de respuesta que van desde "cinco minutos" hasta "un minuto".

Hipótesis nula: Las relaciones de dependencia entre las variables especificadas se ajustarán a los datos observados ya que el estado del conocimiento ha establecido tales relaciones. Sin embargo, las teorías que sustentaron las relaciones de dependencia no explican de un modo integral las asociaciones establecidas.

\section{DISCUSIÓN}


El presente estudio ha demostrado, mediante un modelo estructural, las relaciones causales entre las variables sociodemográficas de sexo y edad sobre la intención de usar Internet. Éste hallazgo es relevante a la luz del estado del arte dada la escasez de estudios al respecto.

Sin embargo, el estado del arte ha demostrado que los programas organizacionales y los software computacionales son los determinantes de las percepciones de eficiencia y utilidad, las actitudes hacia el uso de Internet y la intención de usarla. Las variables sociodemográficas, sexo y edad, podrían incluirse en el TAM considerando dos especificaciones:

La primer especificación de un modelo sería incluyendo al sexo y la edad como variables moderadoras de los programas y software sobre las percepciones, actitudes e intenciones en torno a la predicción del uso de Internet.

La segunda especificación incluiría al sexo y a la edad como variables transmisoras de los programas organizacionales y computacionales sobre las variables psicológicas en torno a la predicción del uso de Internet.

No obstante, el estado del arte parece matizar los efectos de los programas computacionales y organizacionales sobre las variables psicológicas incluyendo otras variables psicológicas tales como el apego, la identidad, la satisfacción o el autocontrol mientras descarta la autoeficiencia computacional, la percepción de utilidad, la actitud hacia el uso de Internet y la intención de uso. Incluso, el estado del arte ha propuesto otros modelos buscando una predicción más especializada de algún software de Internet.

El estado del arte parece dejar en el anonimato las diferencias sexuales, de edad, educativas y salariales que configuran los perfiles de los usuarios de Internet.

Por ello, es indispensable recuperar los perfiles sociodemográficos para incorporarlos en los modelos explicativos de los efectos de los avances e innovaciones de programas computacionales y organizacionales sobre el consumo de productos y servicios en Internet. Incorporar las variables sociodemográficas sería el primer paso para humanizar a Internet y construir una red no sólo de intercambio de datos, sino una de comunicación integral de conocimientos y afectos.

\section{Conclusiones}

Los determinantes del uso de Internet suponen:

- Relaciones de dependencia establecidas a partir de marcos teóricos que explican procesos deliberados, planificados y sistemáticos en torno al procesamiento de información y su incidencia sobre el uso intensivo de tecnologías y dispositivos electrónicos.

- Tales relaciones de dependencia han sido corroboradas empíricamente en diferentes latitudes y con diferentes muestras. Sin embargo, las teorías que 
explican las asociaciones entre cada una de las variables no explican en términos integrales el proceso deliberado que implica el uso de una tecnología.

- En el presente estudio, se contrastó un modelo a partir del cual es posible construir una teoría que explique el proceso de aceptación y adopción de una tecnología o dispositivo electrónico. Empero, se recomienda incluir variables relativas a emociones ya que el uso de Internet está supeditado a relaciones sociales de confianza y compromiso.

\section{REFERENCIAS}

Ademola, R. (2009). Influencia de la ansiedad ante los ordenadores y el conocimiento de su uso en estudiantes de secundaria. Journal of Research in Educational Psychology. 7, 1269-1288

Agarwal, R. \& Venkatesh, V. (2002). Assessing a firm 's web presence: A heuristic evaluation procedure for the measurement of usability. Information System Research. 13, 168-225

Agarwal, R. (2000). Individual acceptance of information technologies. In R. W. Zmud (Ed.) Framing the domains of it management research: glimpsing the future to the past. Pinnaflex, 85-104.

Amoako, K. \& Salam, A. (2004). An extension of the Technology Acceptance Model in an ERP implementation environment. Information \& Management. 41, 731-745

Ballester, R., Gil, M., Gómez, S. y Gil, B. (2010). Propiedades psicométricas de un instrumento de evaluación de la adicción al cibersexo. Psicothema. 22, 1048-1063

Bandura, A. (1977). Self-efficacy: toward a unifying theory of behavioral change. Psychological Review. 84, 191-215

Bandura, A. (1982). Self-efficacy. Mechanism in human agency. American Psychologist. 37, 122-147

Bandura, A. (1993). Perceived self-efficacy in cognitive development and functioning. Educational Psychologist. 28, 117-148

Bandura, A. (1994). Self-efficacy. In V. S. Ramachaudran (ed.). Encyclopedia and Human Behavior. (pp. 71-88). New York: Academic Press

Bandura, A. (1995). Exercise of personal and collective efficacy in changing societies. In A. Bandura (ed.). Self-efficacy in changing societies. (pp. 1-45). New York: Cambriedge University Press.

Bandura, A. (2001). Social cognitive theory: an agentic perspective. Annual Review of Psychology. 52, 1-26 
Barbeite, F. \& Weiss, E. (2004). Computer self ' 'efficacy and anxiety scales for a internet sample: testing measurement equivalence of existing measures and development of new scales. Computers in Human Behaviors. 20, 1-15

Bennett, J., Zimmer, C., Gundlach, M. \& McKnight, H. (2008). Internal and external dimensions of computer self-efficacy: an empirical examination. Transactions on Engineering Management. 55, 628-644

Beranuy, M., Chamarro, A., Graner, C. y Carbonell, J. (2009). Validación de dos escalas breves para evaluar la adicción a Internet y el abuso del móvil. Psicothema. $21,480-485$

Bertrand, M. \& Bouchard, S. (2008). Applying the Technology Acceptance Model to VR with people who are favorable to its use. Journal of Cyber-Therapy \& Rehabilitation. 1, 200-211

Bigne, E., Ruíz, C. \& Sanz, S. (2007). Key drivers of mobile commerce adoption. An exploratory study of Spanish mobile use. Journal of Theoretical and Applied Electronic Commerce Research. 2, 48-60

Bonilla, L. y Bonilla, J. (2006). Estudio comparado de las estimaciones de dos versiones del Modelo de Aceptación de la Tecnología (TAM) mediante los programas AMOS y PLS. Investigaciones Europeas de Dirección y Economía de la Empresa. 12, 95-110

Briñol, P., Horcajo, J., De la Corte, L., Valle, C., Gallardo, I. y Díaz, D. (2004). Efecto de la ambivalencia evaluativa sobre el cambio de actitudes. Psicothema. 16, 373-377

Busch, T. (1995). Geneder differences in self-efficacy and attitudes towards computers Journal of Educational Computers Research. 12, 147-158

Carmel, E. \& Agarwal, R. (2002). The maturation of offshore sourcing of information technology work. MIS Quarterly Executive. 1, 65-77

Compeau, D. \& Higgins, C. (1995). Computer self efficacy: development of measure and initial test. Mis Quarterly. 19, 189-211

Chang, H. (2009). Application of the extended the Technology Acceptance Model to picture archiving and communication systems in dental hospital. Journal of Korean Informatics. 15, 265-272

Chin, W. (2009). A technology acceptance model: mediated and moderated effect. Asia Pacific Management Review. 14, 461-473

Chu, Y. (2006). Levels of computer self efficacy, computer use and earnings in China. Economics Letters. 90, 427-432 
Chuo, Y-H., Tsai, C-H., Lan, Y-L. \& Tsai, C-S. (2011). The effect of organizational support, self efficacy and computer anxiety on the usage intention of e-learning system in hospital. African Journal of Business Management. 5, 5518-5523

D’ambra, J. \& Wilson, C. (2004). Explaining perceived performance of the world wide web; uncertainly and the Task Technology Fit Model. Internet Research. 14, 294-310

Davis F. (1989). Perceived usefulness, perceived ease of use, and user acceptance of information technology. MIS Quarterly. 13, 319-340

Davis, F. \& Venkatesh, V. (1996). A critical assessment of potential measurement biases in the technology acceptance model: three experiments. Journal Computer Studies. 45, 19-45

Davis, F. (1993). User acceptance of information technology: systems, characteristics, user perception and behavioral impacts. International Journal of Man Machine Studies. 8, 475-487

Davis, F. (2006). On the relationship between HCI and technology acceptance research. In P. Zhang \& Galleta, D. (cords.). Human -computer interaction and management information systems: foundations. (pp. 395-401). New York: AMIS

Davis, F., Bagozzi, R. \& Warshaw, P. (1989). User acceptance of computer technology: a comparison of two theoretical models. Management Science. 35, 9821003

Dishaw, M. \& Strong, D. (1999). Extending the Technology Acceptance Model with Task Technology Fit construct. Information \& Management. 36, 9-21

Durndell, A. \& Haag, Z. (2002). Computer self-efficacy, computer anxiety, attitudes toward Internet and reported experience with the Internet, by gender, in an East European sample. Computers in Human Behavior. 18, 521-535

Fuente, A., Herrero, J. y Gracia, E. (2010). Internet y apoyo social: sociabilidad online y ajuste psicosocial en la sociedad de la información. Acción Psicológica. 7, 915

García-Lirios, C. (2007). Percepción de utilidad del comercio electrónico. Enseñanza e Investigación en Psicología, 12, 409-420

García-Lirios, C. (2008). La brecha digital de las generaciones futuras. Entelequía, 8, 61-72

García-Lirios, C. (2011). Estructura de las actitudes hacia el comercio electrónico. Contribuciones a la Economía, 14, 1-10

García-Lirios, C. (2012). Estructura híbrida de los determinantes sociodemográficos 
del consumo electrónico. Gepu, 3, 43-53

García-Lirios, C. (2013). Actitud hacia la utilidad y el riesgo en las redes sociales. Folios, 29, 91-103

Gong, M., Xu, Y. \& Yu, Y. (2004). An enhanced Technology Acceptance Model for web-based learning. Journal of Information Systems Education. 1, 365-375

Ha, S. \& Stoel, L. (2009). Consumer e-shopping acceptance; antecedents in a Technology Acceptance Model. Journal of Business Research. 62, 565-571

Hassan, Y. (2006). Factores de diseño web orientado a la satisfacción y no frustración de uso. Revista Española de Documentación Científica. 29, 239-257

Hee, D. \& McDaniel, S. (2011). Using an extended Technology Acceptance Model in exploring antecedents to adopting fantasy sports league websites. International Journal of Sport Marketing \& Sponsorships. 240-253

Hernández, B., Jiménez, J. y De Hoyos, M. (2007). Aceptación empresarial de las Tecnologías de la Información y Comunicación: un análisis del sector servicios. Revista da Gestao da Tecnologia e Sustemas de Informaçao. 4, 3-22

Hernández, C., Robles, E. y Mella, J. (2009). Medición de la actitud hacia el uso del computador e Internet en estudiantes de enseñanza media en Santiago de Chile. En J. Sánchez (Ed.) Nuevas ideas en informática educativa. (pp. 28-35). Santiago: Universidad de Chile

Herrero, A., Rodríguez, A. y Trespalacios, J. (2006). La adopción del comercio electrónico B2B: una comparación empírica de dos modelos alternativos. Revista Española de Investigación de Marketing. 10, 69-91

Hsu, M. \& Chiu, C. (2004). Internet self efficacy and electronic service acceptance. Decision Support Systems. 38, 369-381

Jiménez, J. y Martín, M. (2007). Indicadores y dimensiones que definen la actitud del consumidor hacia el uso del comercio electrónico. Cuadernos de Economía y Dirección de la Empresa. 31, 7-31

Jonhson, D. \& Warldlow, J. (2004). Computers experiences, self efficacy and knowledge of undergraduate student entering a land grant college of agriculture by year and gender. Journal of Agricultural Education. 45, 53-64

Khorrami, O. (2001). Researching computer self-efficacy. International Educational Journal. 2, 17-26

Klopping, I. \& McKinney, E. (2004). Extending the Technology Acceptance Model and the Task Technology Fit Model to Consumer e-commerce. Information Technology, 
Lai, V. \& Lee, H. (2005). Technology acceptance model for Internet banking: an invariance analysis. Information \& Management. 42, 373-386

Lee, D., Park, J. \& Ahn, J. (2001). On the explanation of factors affecting e commerce adoption. Twenty Second International Conference on Information System. Paper 14

Liang, J., \& Tsai, C. (2008). Internet self-efficacy and preferences toward constructivist Internet-based learning environments: A study of pre-school teachers in Taiwan. Educational Technology \& Society, 11 (1), 226-237.

Limayen, M., Hirt, S. \& Cheung, C. (2007). How habit limits the predictive power of intention: the case of information systems continuance. Mis Quarterly. 31, 705-737

Liu, S., Liao, H. \& Peng, C. (2005). Applying the Technology Acceptance Model and flow theory to online e-learning user's acceptance behavior. Issues in Information Systems. 2, 175-182

López, L. y López, J. (2011). Los modelos de adopción de tecnologías de información desde el paradigma actitudinal. Cadernos Ebape. 9, 176-196

Lorenzo, C., Constantinides, E., Gómez, E. y Geurts, P. (2008). Análisis del consumo virtual bajo la influencia de las dimensiones constituyentes de la experiencia web. Estudios sobre Consumo. 84, 53-65

Mafé, R. y Blas, S. (2007). Influencia del perfil sociodemográfico, relaciones con el medio y actitud ante la compra a distancia en el comportamiento de compra por televisión. Investigaciones Europeas de Dirección y Economía de la Empresa. 13, 131-143

Martínez, J. y De Hoyos, M. (2007). Indicadores y dimensiones que definen la actitud del consumidor hacia el uso del comercio electrónico. Cuadernos de Economía y Dirección de la Empresa. 31, 7-30

Meléndez, P. y Moreno, G. (2006). El uso de tecnologías basadas en Internet para el aprendizaje. Un estudio exploratorio en el contexto del Modelo de la Aceptación de la Tecnología. Revista Europea de Dirección y Economía de la Empresa. 12, 217-230

Montero, Y. (2006). Factores de diseño web orientado a la satisfacción y no frustración de uso. Revista Española de Documentación Científica. 29, 239-257

Orantes, S. (2011). Viabilidad del Modelo de la Aceptación de la Tecnología en las empresas mexicanas. Una aproximación a las actitudes y percepciones de los usuarios de las tecnologías de la información. Revista Digital Universitaria. 12, 1-15 
Paraskeva, F., Bouta, H. \& Papagianni, A. (2008). Individual characteristics and computer self-efficacy in secondary education teachers to integrated technology in education practice. Computers \& Education. 50, 1084-1091

Pin, H. (2004). Extended technology acceptance model of Internet utilization behavior. Information and Management. 4, 719-729

Porter, C. \& Donthu, N. (2006). Using the Technology Acceptance Model to explain how attitudes determine Internet usage: the role of perceived access barriers and demographics. Journal of Business Research. 59, 999-1007

Porter, S. (2006). Using the technology acceptance model to explain how attitudes determine internet usage: the role perceived access barriers and demographic. Journal of Business Research. 59, 999-1007

Reed, E. \& Wolniak, G. (2005). Diagnosis or determination: assessment explained through Human Capital Theory and the concept of aptitudes. Journal of Sociology. 1, $1-5$

Reid, M. \& Levy, Y. (2008). Integrating trust and computer self-efficacy with TAM: an empirical assessment of customers' acceptance of banking information system. Journal of Internet Banking and Commerce. 12, 1-18

Roca, J., Chiu, C. \& Martínez, F. (2006). Understanding e-learning continuance intention: an extension of the Technology Acceptance Model. International Journal of Human Computer Studies. 64, 683-696

Rose, J. \& Fogarty, G. (2006). Determinants of perceived usefulness and perceived ease of use in the technology acceptance model: senior consumer adoption of selfservice banking technologies. Marketing \& Management Development. 2, 122-130

Ruíz, C. y García, J. (2007). Factores determinantes de la decisión de compra en Internet. Un análisis de la formación a distancia. Estudios sobre Consumo. 80, 49-60

Ruíz, C. y Sanz, S. (2007). Influencia del perfil sociodemográfico, relaciones con el medio y actitud ante la compra a distancia en el comportamiento de compra por televisión. Investigaciones Europeas de Dirección y Economía de la Empresa. 13, 131-143

Saadé, R. \& Kira, D. (2009). Computer anxiety in e-learning: the effect of computer self-efficacy. Journal of Information Technology Education. 8, 1-15

Saadé, R., Nebebe, F. \& Tan, W. (2007). Viability of the Tecnology Acceptance Model in multimedia learning environments: a comparative studies. Interdisciplinary Journal of Knowledge and Learning Objects. 3, 1-10

Sam, H., Othman, A. , \& Nordin, Z. (2005). Computer Self-Efficacy, Computer 
Anxiety, and Attitudes toward the Internet: A Study among Undergraduates in Unimas. Educational Technology \& Society, 8 (4), 205-219.

Sandoval, R. y Saucedo, N. (2010). Grupos de interés en las redes sociales: el caso de Hi5 y Facebook en México. Educación y Humanidades. 4, 132-142

Sanz, S., Mafé, C. y Aldás, J. (2008). La influencia de la dependencia en el comercio electrónico B2C. Propuesta de un modelo integrador aplicado a la intención de compra futura en Internet. Cuadernos de Economía y Dirección de la Empresa.36, 45-76

Shepers, J. \& Wetzels, M. (2007). A meta-analysis of the Technology Acceptance Model: investigating subjective norm and moderation effects. Information \& Management. 47, 90-110

Shih, H. (2004). Extended Technology Acceptance Model of Internet utilization behavior. Information \& Management. 41, 719-729

Shrrof, R., Denenn, C. \& Ng, E. (2011). Analysis of the Technology Acceptance Model in examining student's behavioral intention to use an e-portfolio system. Australasian Journal of Educational Technology. 27, 600-618

Simsek, A. (2011). The relationships between computer anxiety and computer self efficacy. Contemporary Educational Technology. 2, 177-187

Su, L., Hsiu, L. y Cheng, P. (2005). Applying the Technology Acceptance Model and flow theory to online e-learning user's acceptance behavior. Issues in Information System. 6, 175-182

Suárez, A., García, N. y Álvarez, M. (2008). Actitud de los consumidores hacia encuestas on-line. Estudios sobre Consumo. 84, 1-17

Teh, P-L., Chong, C-W., Yong, C-C. \& Yew, S-Y. (2010). Internet self-efficacy, computer self-efficacy, and cultural factor on knowledge sharing behavior. African Journal of Business Management. 4, 4086-4095

Torkzadeh, G., Cha, J. \& Demirhan, D. (2006). A contingency model of computer and internet self efficacy. Information \& Management. 43, 541-550

Vekiri, I. \& Chronaki, A. (2008). Gender issues in technology use: perceived social support, computer self-efficacy and value beliefs, and computer beyond school. Computers \& Education. 51, 1392-1404

Venkatesh, V., Morris, M., Davis, G. \& Davis, F. (2003). User acceptance of information technology: toward a unified view. Mis Quarterly. 27, 425-476

Wai, W., Andersson, R. y Oslear, K. (2005). Examining user acceptance of computer 
technology: an empirical study of student teachers. Journal of Computer Assisted Learning. 21, 387-395

Winocur, R. (2006). Internet en la vida cotidiana de los jóvenes. Revista Mexicana de Sociología. $68,551-580$

Wu, J., Shen, W., Lin, L., Greenes, R. \& Bates, D. (2008). Testing the Technology Acceptance Model for evaluating healthcare professionals intention to use and adverse event reporting system. International Journal for Quality in Health Care. 20, 123-129

Wu, Y. \& Tsai, C. (2006). University students Internet attitudes and self efficacy: a study at tree universities in Taiwan. Cyberpsychology \& Behavior. 9, 441-452

Zhangxi, L., Binjia, S. y Linhua, Y. (2007). Understanding Internet banking: an empirical investigation of potential customers' acceptance in Mailand, China. American Conference of Information System. 485, 1-14 